\title{
Hot and dry: how plants can thrive in future climates
}

\author{
Manzer H. Siddiqui ${ }^{1} \cdot$ M. Nasir Khan ${ }^{2} \cdot$ Vijay Pratap Singh ${ }^{3}$
}

Received: 27 December 2021 / Accepted: 27 December 2021 / Published online: 17 February 2022

(c) The Author(s), under exclusive licence to Springer-Verlag GmbH Germany, part of Springer Nature 2022

Increased global temperature is the hallmark of climate change. Higher air temperature creates heat stress (HS) that adversely affects plants at morphological, cellular, subcellular and molecular levels. The detrimental effect of HS combined with drought stress (DS) further aggravates the conditions that suppress key metabolic processes including photosynthesis, primary and secondary metabolism, and signaling. Sustained exposure of plants to HS and DS induces the generation of reactive oxygen species (ROS) which, at a lower concentration, perform the function of signaling, however, higher levels of ROS creates oxidative stress and damages macromolecules (Piacentini et al. 2020), and inactivates and destroys proteins (Liu et al. 2010). Plants have various types of enzymatic and non-enzymatic antioxidant defense systems, which enable them to maintain redox homeostasis and functional cellular conditions (Foyer and Noctor 2011). In addition, the onset of HS and DS also induces the expression of heat shock proteins, heat shock factors, plant growth regulators, ion transporters and factors involved in signaling cascades and transcriptional controllers, which collectively are essential to counteract HS and DS. However, the mechanism behind the symphonic operation of these defense systems is a long way from being completely deciphered.

In this special issue, we look back on recent advances in understanding the physiological and biochemical mechanisms involved in plant adaptation and resistance to HS and DS. We also look into the future of stress tolerance mechanisms of HS and DS by advanced molecular techniques.

Manzer H. Siddiqui

mhsiddiqui@ksu.edu.sa

1 Department of Botany and Microbiology, College of Science, King Saud University, Riyadh 11451, Saudi Arabia

2 Department of Biology, College of Haql, University of Tabuk, Tabuk, Saudi Arabia

3 Plant Physiology Laboratory, Department of Botany, C.M.P. Degree College, A Constituent Post Graduate College of University of Allahabad, Prayagraj 211002, India
The review article by Lal et al. (2021) discusses the role of the interplay of antioxidants and hormones in imparting heat stress tolerance in wheat and also discusses approaches to develop heat-tolerant wheat varieties. Riyazuddin et al. (2021) summarize the role of dehydrin (DHN) proteins. They describe the mechanism of DHNs-mediated DS tolerance in plants and their interaction with several phytohormones. The role of calcium-dependent protein kinase (CDPK) in the salt stressed-potato was studied by Grossi et al. (2021). The CDPKs are sensor-transducers that decode $\mathrm{Ca}^{2+}$ signatures triggered by abiotic stimuli and translate them into physiological responses. The results of the study show that the tolerant plants that overexpressed StCDPK2 have a more efficient antioxidant system than the wild-type plants when exposed to salt stress (Grossi et al. 2021). Rashid et al. (2021) studied the responses of wheat plants that were inoculated with drought-tolerant plant growth-promoting rhizobacteria (PGPR) Bacillus megaterium (MU2) and Bacillus licheniformis (MU8) under induced drought stress. Verma et al. (2021) performed the characterization of ascorbate peroxidase (APX) gene family in two Brassica species (Brassica juncea and Brassica rapa) for tolerance against abiotic stresses. They identified $16 B j u A P X$ and 9 $B r a A P X$ genes and $2 A P X-R$ genes each in $B$. juncea and $B$. rapa genomes, respectively Verma et al. (2021). Balfagón et al. (2021) explored the significance of the rootstock on scion performance and antioxidant response to improve crop performance and maintain citrus yield under drought and heat stress conditions. Lone et al. (2021) found that exogenous brassinosteroid and jasmonic acid improved drought tolerance in $B$. rapa genotypes by regulating osmolytes, antioxidants and photosynthetic systems. The study performed by Raja et al. (2021) developed the ascorbate-glutathione (AsA-GSH) overexpression lines by stacking the genes of the AsA-GSH pathway genes isolated from Pennisetum glaucoma under stress-inducible promoter RD29A. The exposure of transgenic seedlings of $P$. glaucoma to varying stress regiments exhibited an escalation in the antioxidant enzyme activity and stress tolerance (Raja et al. 2021). Madhu et al. (2021) identified seven glutathione reductase 
(GR) genes in wheat (Triticum aestivum) for comparative structural characterization, phylogenetic analysis and expression profiling with the GR genes of other cereal plants. The differential expression of $T a G R$ genes and enhanced GR enzyme activity have important functions under drought, heat, salt and arsenic stress. Kumar et al. (2021) reviewed the involvement of sugar metabolism during pre- and postfertilization events in plants under high temperature stress. They also discussed the synergistic or antagonistic interactions among sugars, hormones and reactive oxygen species at various points of sucrose flow from source to sink under high temperature stress. The review article by Poór et al. (2021) discusses the recent studies of ethylene involvement in plant responses to heat stress and its functional regulation, and molecular mechanism underlying the plant responses in the mitigation of heat-induced damages. Review articles by Bhardwaj et al. (2021) and Raza (2020) shed light on the role of -omics approaches in enhancing drought and heat stress tolerance in plants. Whereas, Singh et al. (2021) and Mishra et al. (2021) discussed the regulatory role of small RNAs in modulating high temperature and drought stress responsiveness in plants, respectively. The last four review articles of this special issue are dedicated to the molecular approaches of stress tolerance and adaptive responses of plants to various abiotic stresses. The review article by Haider et al. (2021a, b) and Saini et al. (2021) appraises the potential of emerging molecular technologies in improving thermotolerance in plants. Chennakesavulu et al. (2021) provides a widespread overview of the CRISPR/Cas genome editing technologies that can be used for obtaining cultivars with enhanced tolerance to various abiotic stress factors.

The diverse articles published in this special issue provide an in-depth comprehension of drought and heat stress challenges faced by crop plants. The findings and conclusions of these studies-ranging from physiological to cellular, sub-cellular and molecular-will assist in designing new crop varieties with the intrinsic capacity to survive under challenging climatic conditions.

Funding Authors received no funding for the work reported here.

\section{Declarations}

Conflict of interest The authors declare that they have no competing interests.

\section{References}

Balfagón D, Terán F, de Oliveira T et al (2021) Citrus rootstocks modify scion antioxidant system under drought and heat stress combination. Plant Cell Rep. https://doi.org/10.1007/ s00299-021-02744-y

Bhardwaj A, Devi P, Chaudhary S et al (2021) 'Omics' approaches in developing combined drought and heat tolerance in food crops. Plant Cell Rep. https://doi.org/10.1007/s00299-021-02742-0

Chennakesavulu K, Singh H, Trivedi PK et al (2021) State-of-theart in CRISPR technology and engineering drought, salinity, and thermo-tolerant crop plants. Plant Cell Rep. https://doi.org/10. 1007/s00299-021-02681-w

Foyer CH, Noctor G (2011) Ascorbate and glutathione: the heart of the redox hub. Plant Physiol 155:2-18. https://doi.org/10.1104/ pp. 110.167569

Grossi CEM, Santin F, Quintana SA et al (2021) Calcium-dependent protein kinase 2 plays a positive role in the salt stress response in potato. Plant Cell Rep. https://doi.org/10.1007/ s00299-021-02676-7

Haider S, Iqbal J, Naseer S et al (2021a) Molecular mechanisms of plant tolerance to heat stress: current landscape and future perspectives. Plant Cell Rep 40:2247-2271. https://doi.org/10.1007/ s00299-021-02696-3

Haider S, Iqbal J, Naseer S et al (2021b) Unfolding molecular switches in plant heat stress resistance: a comprehensive review. Plant Cell Rep. https://doi.org/10.1007/s00299-021-02754-w

Kumar S, Thakur M, Mitra R et al (2021) Sugar metabolism during pre- and post-fertilization events in plants under high temperature stress. Plant Cell Rep. https://doi.org/10.1007/ s00299-021-02795-1

Lal MK, Tiwari RK, Gahlaut V et al (2021) Physiological and molecular insights on wheat responses to heat stress. Plant Cell Rep. https://doi.org/10.1007/s00299-021-02784-4

Liu X, Peng K, Wang A et al (2010) Cadmium accumulation and distribution in populations of Phytolacca americana L. and the role of transpiration. Chemosphere 78:1136-1141. https://doi.org/10. 1016/j.chemosphere.2009.12.030

Lone AW, Majeed N, Yaqoob U et al (2021) Exogenous brassinosteroid and jasmonic acid improve drought tolerance in Brassica rapa L. genotypes by modulating osmolytes, antioxidants and photosynthetic system. Plant Cell Rep. https://doi.org/10.1007/ s00299-021-02763-9

Madhu KA, Tyagi S et al (2021) Exploration of glutathione reductase for abiotic stress response in bread wheat (Triticum aestivum L.). Plant Cell Rep. https://doi.org/10.1007/s00299-021-02717-1

Mishra S, Sahu G, Shaw BP (2021) Integrative small RNA and transcriptome analysis provides insight into key role of miR408 towards drought tolerance response in cowpea. Plant Cell Rep. https://doi.org/10.1007/s00299-021-02783-5

Piacentini D, Rovere FD, Sofo A et al (2020) Nitric oxide cooperates with auxin to mitigate the alterations in the root system caused by cadmium and arsenic. Front Plant Sci 11:1182. https://doi.org/10. 3389/fpls.2020.01182

Poór P, Nawaz K, Gupta R et al (2021) Ethylene involvement in the regulation of heat stress tolerance in plants. Plant Cell Rep. https:// doi.org/10.1007/s00299-021-02675-8

Raja V, Wani UM, Wani ZA et al (2021) Pyramiding ascorbate-glutathione pathway in Lycopersicum esculentum confers tolerance to drought and salinity stress. Plant Cell Rep. https://doi.org/10. 1007/s00299-021-02764-8

Rashid U, Yasmin H, Hassan MN et al (2021) Drought-tolerant Bacillus megaterium isolated from semi-arid conditions induces systemic tolerance of wheat under drought conditions. Plant Cell Rep. https://doi.org/10.1007/s00299-020-02640-x

Raza A (2020) Metabolomics: a systems biology approach for enhancing heat stress tolerance in plants. Plant Cell Rep. https://doi.org/ 10.1007/s00299-020-02635-8

Riyazuddin R, Nisha N, Singh K et al (2021) Involvement of dehydrin proteins in mitigating the negative effects of drought 
stress in plants. Plant Cell Rep. https://doi.org/10.1007/ s00299-021-02720-6

Saini N, Nikalje GC, Zargar SM et al (2021) Molecular insights into sensing, regulation and improving of heat tolerance in plants. Plant Cell Rep. https://doi.org/10.1007/s00299-021-02793-3

Singh RK, Prasad A, Maurya J et al (2021) Regulation of small RNAmediated high temperature stress responses in crop plants. Plant Cell Rep. https://doi.org/10.1007/s00299-021-02745-x

Verma D, Upadhyay SK, Singh K (2021) Characterization of APX and APX-R gene family in Brassica juncea and B. rapa for tolerance against abiotic stresses. Plant Cell Rep. https://doi.org/10.1007/ s00299-021-02726-0

Publisher's Note Springer Nature remains neutral with regard to jurisdictional claims in published maps and institutional affiliations. 